\title{
Optimal Torque and Stiffness Control in Compliantly Actuated Robots
}

\author{
D. J. Braun ${ }^{*, \dagger}$, F. Petit ${ }^{\ddagger}$, F. Huber ${ }^{\ddagger}$, S. Haddadin ${ }^{\ddagger}$, P. van der Smagt ${ }^{\ddagger}, \S$, A. Albu-Schäffer ${ }^{\ddagger}$ and S. Vijayakumar ${ }^{\dagger}$
}

\begin{abstract}
Anthropomorphic robots that aim to approach human performance agility and efficiency are typically highly redundant not only in their kinematics but also in actuation. Variable-impedance actuators, used to drive many of these devices, are capable of modulating torque and passive impedance (stiffness and/or damping) simultaneously and independently. Here, we propose a framework for simultaneous optimisation of torque and impedance (stiffness) profiles in order to optimise task performance, tuned to the complex hardware and incorporating real-world constraints. Simulation and hardware experiments validate the viability of this approach to complex, state dependent constraints and demonstrate task performance benefits of optimal temporal impedance modulation.
\end{abstract}

Index Terms-Variable-stiffness actuation, physical constraints, optimal control.

\section{INTRODUCTION}

Modern anthropomorphic robots, designed to mimic human behaviour and performance, are highly redundant in their kinematics and also actuation (e.g., DLR Hand-Arm System) [1]. Variable impedance actuators (VIAs), used to drive these complex devices, are capable of simultaneous torque and passive impedance (stiffness and damping) modulation, as opposed to classic torque controlled designs. To perform impedance modulation [2], VIAs often employ multiple motors per joint [3]-[8] in contrast to a single one on a torque-controlled device. However, the benefit of including more motor units does not come for free. Indeed, VIAs are highly nonlinear and assert numerous constraints: range rate and effort limitations that are often neglected on classical devices. Due to these reasons, in many cases the benefit of variable-impedance control can only be shown if the control problem is algorithmically treated and if the control redundancy is optimally resolved in a task specific manner [9]-[12].

In this paper we employ non-linear optimal control to resolve the actuator redundancy, realising variable stiffness control tuned to the task and specificity of the system dynamics. The proposed approach can be used to embed bandwidth limitations of the actuators and deformation limits on the compliant elements into the formulation. This formulation is tested on two conceptually different variable-stiffness systems both realised in hardware. Its viability for practical applications is demonstrated by a ball-throwing experiment implemented on the DLR Hand-Arm System (Fig. 1).

\footnotetext{
${ }^{\dagger}$ Institute of Perception Action and Behaviour, University of Edinburgh, United Kingdom.

${ }^{\ddagger}$ German Aerospace Centre (DLR), Institute of Robotics and Mechatronics, 82234 Oberpfaffenhofen-Wessling, Germany.

$\S$ Institute of Informatics, Technische Universität München.

*Adress correspondence to: DJB, E-mail: david.braun@ed.ac.uk.
}

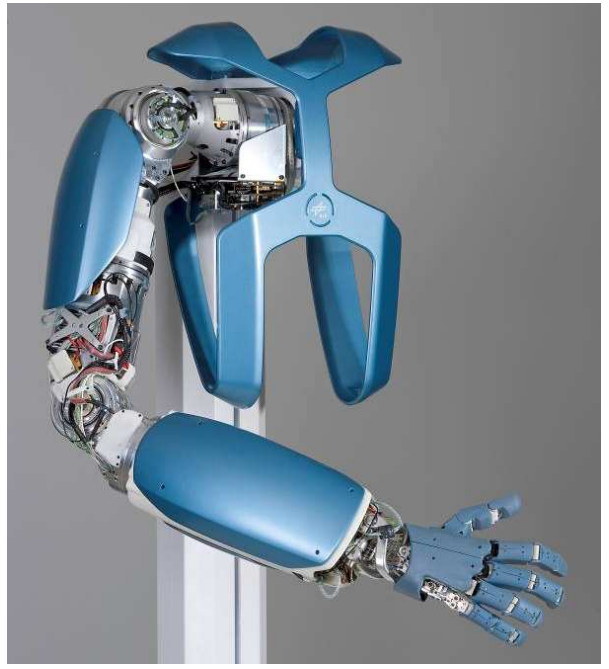

Fig. 1. DLR Hand-Arm System.

\section{MOdELING COMPLIANTLY ACTUATED SYSTEMS}

We begin by discussing the dynamics model for a compliantly actuated robotic system capable of simultaneous torque and passive stiffness modulation in a feed-forward fashion ${ }^{1}$. We will specifically look at the formalisation of: 1) rigid body dynamics (i.e., link-side dynamics) and the motorside dynamics of the robot, 2) static torque and stiffness characteristics of the compliant mechanism that is in-built in the actuators and 3) the physical constraints imposed by the bandwidth limitations of the motors and the range limitations inherent to many VSA designs.

\section{A. Link-side and motor-side dynamics}

Consider an $n$ degree-of-freedom robotic system, the configuration of which is uniquely specified by $\mathbf{q} \in \mathbb{R}^{n}$ generalized coordinates (e.g., joint angles). Let this system be equipped with $m$ compliant actuators (e.g., SEAs and/or VSAs), and let $\boldsymbol{\theta} \in \mathbb{R}^{m}$ denote the motor angles reflected through gear reduction, see Fig. 2. In the following we

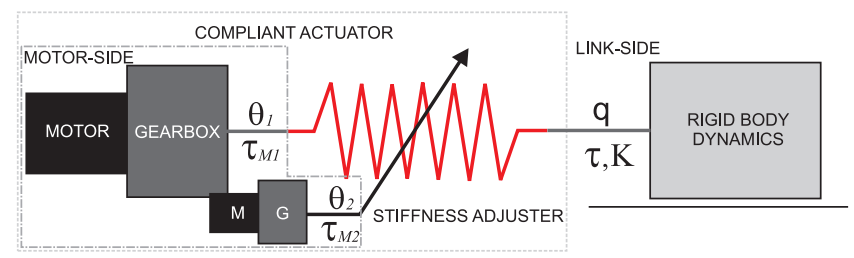

Fig. 2. Schematic representation of a variable stiffness system.

\footnotetext{
${ }^{1}$ This is unlike active stiffness control (e.g., [13]) realised through position
} feedback on torque controllable devices. 
define the kinetic and the gravitational potential energy of the rigid body dynamics ${ }^{2}: \mathcal{T}=\mathcal{T}(\mathbf{q}, \dot{\mathbf{q}}), \mathcal{U}_{G}=\mathcal{U}_{G}(\mathbf{q})$ and the elastic potential energy of the compliant actuators ${ }^{3}$ $\mathcal{U}_{E}=\mathcal{U}_{E}(\mathbf{q}, \boldsymbol{\theta})$, to derive the link and motor-side equations of motion ${ }^{4}$ through application of the Lagrangian formalism [15],

$$
\begin{array}{r}
\mathbf{M}(\mathbf{q}) \ddot{\mathbf{q}}+\mathbf{C}(\mathbf{q}, \dot{\mathbf{q}}) \dot{\mathbf{q}}+\mathbf{G}(\mathbf{q})=\boldsymbol{\tau}(\mathbf{q}, \boldsymbol{\theta}), \\
\mathbf{J} \ddot{\boldsymbol{\theta}}+\mathbf{B} \dot{\boldsymbol{\theta}}=\boldsymbol{\tau}_{E}(\mathbf{q}, \boldsymbol{\theta})+\boldsymbol{\tau}_{M},
\end{array}
$$

where $\mathbf{M}=\partial^{2} \mathcal{T} / \partial \dot{\mathbf{q}}^{2} \in \mathbb{R}^{n \times n}$ is the symmetric and positive definite mass matrix, $\mathbf{C} \dot{\mathbf{q}}=\left(\partial^{2} \mathcal{T} / \partial \dot{\mathbf{q}} \partial \mathbf{q}\right) \dot{\mathbf{q}}-\partial \mathcal{T} / \partial \mathbf{q} \in \mathbb{R}^{n}$ are the Coriolis and normal inertial forces, $\mathbf{G}=\partial \mathcal{U}_{G} / \partial \mathbf{q} \in$ $\mathbb{R}^{n}$ represents the gravitational forces, $\boldsymbol{\tau}=-\partial \mathcal{U}_{E} / \partial \mathbf{q} \in \mathbb{R}^{n}$ are the elastic joint torques delivered by the VS actuators to the link-side dynamics, $\mathbf{J} \in \mathbb{R}^{m \times m}$ is the rotational inertia of the motor-gearbox assembly, $\mathbf{B} \in \mathbb{R}^{m \times m}$ represents the intrinsic viscous damping ${ }^{5}, \boldsymbol{\tau}_{E}=-\partial \mathcal{U}_{E} / \partial \boldsymbol{\theta} \in \mathbb{R}^{m}$ is the reaction torque by the compliant elements while $\tau_{M} \in \mathbb{R}^{m}$ is the motor torque (reflected through the gear reduction). Control of $\boldsymbol{\theta}$ is realised with a PD control law ${ }^{6}$,

$$
\boldsymbol{\tau}_{M}=-\mathbf{K}_{M}\left(\boldsymbol{\theta}-\boldsymbol{\theta}_{d}\right)-\mathbf{B}_{M} \dot{\boldsymbol{\theta}},
$$

where $\mathbf{K}_{M} \in \mathbb{R}^{m \times m}$ and $\mathbf{B}_{M} \in \mathbb{R}^{m \times m}$ contain the servo gains and $\boldsymbol{\theta}_{d} \in \mathbb{R}^{m}$ is the desired position. An ideal closedloop motor response of (2) and (3) is critically damped and infinitely fast. Practically this requires large stiffness gain $\mathbf{K}_{M} \gg 1$ (with adequate damping) and would ensure that the motors are position controllable: $\boldsymbol{\theta} \approx \boldsymbol{\theta}_{d}$. In general however, highly-geared actuation puts severe restriction on the motor velocities/accelerations Due to this reason, a rapidly changing desired trajectory $\boldsymbol{\theta}_{d}$ that does not respect the bandwidth limitation of the closed loop motor dynamics will be poorly tracked. In Section III, we provide a simple formulation to compute desired optimal trajectories that avoids this issue.

\section{B. Static torque/stiffness characteristics of the actuators}

According to (1), using the motor side positions $\boldsymbol{\theta}$ as inputs,

$$
\boldsymbol{\tau}=\boldsymbol{\tau}(\mathbf{q}, \boldsymbol{\theta})
$$

provides the elastic joint torques as actuator outputs. The state dependence of this static torque characteristic is due to the passive elasticity (in-built in the actuators) and not due to position feedback. Under feed-forward motor side

\footnotetext{
${ }^{2}$ The kinetic and the potential energy calculated here should include the actuators as rigid objects attached to the robot.

${ }^{3}$ Here we assume that the actuator compliance is generated by linear and/or nonlinear springs with no restriction on generality.

${ }^{4}$ Note that the motor-side model (2) represents the dynamics for all the motors $m \geq n$ used in the VS actuators (including the motors for stiffness adjustment), see Fig.2. For elastic joint robots this model reduces to the one proposed in [14] where $m=n$ and $\boldsymbol{\tau}_{E}=-\boldsymbol{\tau}$.

${ }^{5}$ The damping term in (2) can be seen as an approximation of the real frictional effects.

${ }^{6}$ It is important to note that using motor-side servo control does not limit the bandwidth of the link-side motion on compliantly actuated systems. This is because the intrinsic compliance in the actuator effectively decouples the link-side and the motor-side dynamics. Such decoupling is one of the main reasons why exploitation of the link-side dynamics is not prevented with high-gain motor servo control.
}

control, $\boldsymbol{\theta}=\boldsymbol{\theta}(t)$, this passive elasticity, reflected on the joint defines the output stiffness of the actuators. By definition, this stiffness relates the link-side torque response to the associated position perturbation:

$$
\mathbf{K}=-\partial \boldsymbol{\tau}(\mathbf{q}, \boldsymbol{\theta}) / \partial \mathbf{q},
$$

where $^{7} \mathbf{K}=\mathbf{K}(\mathbf{q}, \boldsymbol{\theta}) \in \mathbb{R}^{n \times n}$. By setting a constant motor-side positions, (4) and (5) define the passive torque and stiffness characteristics of the actuators. Using a timedependent motor positions, these passive characteristics can be simultaneously and continuously modulated, although they may not be independent. The ability to change $\boldsymbol{\tau}(\mathbf{q}, \boldsymbol{\theta})$ and $\mathbf{K}(\mathbf{q}, \boldsymbol{\theta})$ independently is however one of the main attributes of a variable-stiffness actuator. In this light, we now turn to define two analytical conditions that are both necessary and sufficient for simultaneous and independent torque and stiffness modulation: for all admissible $(\mathbf{q}, \boldsymbol{\theta})$,

$$
\begin{array}{r}
\operatorname{det}\left(\left[\frac{\partial \boldsymbol{\tau}}{\partial \boldsymbol{\theta}}\right]\left[\frac{\partial \boldsymbol{\tau}}{\partial \boldsymbol{\theta}}\right]^{+}\right) \neq 0, \\
\operatorname{rank}\left(\left[\frac{\partial \mathbf{k}}{\partial \boldsymbol{\theta}}\right]\left(\mathbf{I}-\left[\frac{\partial \boldsymbol{\tau}}{\partial \boldsymbol{\theta}}\right]^{+}\left[\frac{\partial \boldsymbol{\tau}}{\partial \boldsymbol{\theta}}\right]\right)\right) \neq 0,
\end{array}
$$

where $\mathbf{k}=\operatorname{vec}(\mathbf{K}), \mathbf{I} \in \mathbb{R}^{m \times m}$ is an identity matrix while $(*)^{+}$denotes the Moore-Penrose generalized inverse of $(*)$. The first condition (6) allows all components of the actuators' torque to be affected through $\boldsymbol{\theta}$ while the second condition (7) ensures that motor positions that leave the joint torques unaffected can be used for stiffness modulation. Moreover, the number of elements in the stiffness matrix that can be modulated is defined by the rank in (7). In summary, (6) and (7) make independent torque/stiffness modulation viable ${ }^{8}$.

\section{Physical constraints during variable-stiffness control}

Variable stiffness devices often posses state inequality constraints due to:

1) range limits on the motor positions and also bandwidth limitation (represented by rate constraints) posed by the servo-controlled motor dynamics,

$$
\boldsymbol{\theta}_{m} \preceq \boldsymbol{\theta} \preceq \boldsymbol{\theta}_{M},|\dot{\boldsymbol{\theta}}| \preceq \dot{\boldsymbol{\theta}}_{M},
$$

2) deformation limits of the compliant elements (i.e., springs in-built in the actuators)

$$
\boldsymbol{\phi}_{m}(\boldsymbol{\theta}) \preceq \phi(\mathbf{q}, \boldsymbol{\theta}) \preceq \phi_{M}(\boldsymbol{\theta}),
$$

where $\boldsymbol{\theta}_{m} \prec \boldsymbol{\theta}_{M}$ and $\phi_{m} \prec \phi_{M}$ denote the constraint boundaries while $\dot{\boldsymbol{\theta}}_{M}$ and $\ddot{\boldsymbol{\theta}}_{M}$ defines the maximal velocities that can be achieved by the motors.

In the next section, we provide a simple formulation that allows (8) and (9) to be enforced as hard constraints (i.e.,

\footnotetext{
${ }^{7}$ Off-diagonal elements in the stiffness matrix would appear if we use actuators where configuration change on one joint induces torque change on another joint (in a human arm model [16] this is achieved through biarticular muscles).

${ }^{8}$ In practice, VSA may have singular regions for some $(\mathbf{q}, \boldsymbol{\theta}) \in \mathbb{R}^{n} \times \mathbb{R}^{m}$ where simultaneous torque and stiffness modulation cannot be achieved, but as long as these are isolated and are restricted to a small portion of the work-space, practical issues are not expected.
} 
constraints that cannot be violated) during the optimal control planning. These constraints are particularly important for realising physically viable plans. This may be especially the case for the deformation limits (9), since violation of these constraints would permanently damage the VS mechanism.

\section{OPTIMAL VARIABLE-STIFFNESS CONTROL}

In this section, we develop a paradigm to realise optimal variable-stiffness control that includes the model of the system dynamics together with all the relevant constraints required for physically realizable control planning. Using a non-linear optimal control framework, the proposed formulation is then employed to devise the inputs for variablestiffness modulation that is best suited to the task considered.

\section{A. The role of constraints}

1) Motivation: In Section II-C, we have outlined fundamental constraints that may be applicable during variable stiffness control. Incorporating these constraints (in full or in part) is necessary to make the results applicable to real-world problems. There is however also another equally important reason why the effect of these constraints must be considered once variable stiffness control is required. This is because variable stiffness control strategies may qualitatively differ depending on range/bandwidth limitations of the actuators. To illustrate this point by an example, let us consider two dynamical systems performing a task where rapid stiffness variation is beneficial (e.g., bang-bang control is optimal). Let us now assume that due to bandwidth limitations of the actuators, one of the systems is not able to change its stiffness fast enough to realise a bang-bang strategy that would lead to improved task performance. Under such condition, the optimal strategy would be smoothed out to an averaged impedance. This hypothetical example, evidenced through our numerical investigations (exemplified in Section IV), motivates the treatment of constraints detailed in this section.

2) Basic idea: While incorporating all the relevant constraints into the optimisation is vital, efficient numerical treatment of them is non-trivial. Indeed, solving an optimal control problem with control and state inequality constraints usually requires a computationally demanding non-linear constrained optimisation algorithm. Moreover, excessive application of constraints often make the associated formulation more susceptible to local-minima issues. Finding an optimal solution to such a formulation is difficult. Therefore, reduction of the number/complexity of the constraints is preferred. In the next section we address this problem by replacing state inequality constraints with control constraints that are easier to enforce numerically.

\section{B. Embedding state inequality constraints through control constraints}

Let us now formally define the admissible set of states as

$$
\begin{aligned}
\Psi=\left\{\boldsymbol{\psi} \in \mathbb{C}^{2}\left([0, T], \mathbb{R}^{m}\right):\right. \\
\left.\boldsymbol{\psi}_{m}(\boldsymbol{\theta}) \preceq \boldsymbol{\psi}(\mathbf{q}, \boldsymbol{\theta}) \preceq \boldsymbol{\psi}_{M}(\boldsymbol{\theta})\right\},
\end{aligned}
$$

where $\psi$ denotes the set of generalized quantities $\psi \in \mathbb{R}^{m}$ (e.g., motor position, spring length) to be constrained during the motion while $\boldsymbol{\psi}_{m} \prec \boldsymbol{\psi}_{M}$ are the associated (possibly state-dependent) lower and upper bounds. Now, by choosing: $\boldsymbol{\psi}=\boldsymbol{\theta}$ we can represent (8) while by using $\boldsymbol{\psi}=\boldsymbol{\phi}(\mathbf{q}, \boldsymbol{\theta})$, the constraints of (9) can be expressed. In this light, (10) defines a general representation of state inequality constraints to be enforced during the motion. Next we proceed to explicitly embed (10) into the formulation using control constraints. For this purpose, we introduce:

1) a canonical control set ${ }^{9}$

$$
U=\left\{\mathbf{u} \in P C\left([0, T], \mathbb{R}^{m}\right): \mathbf{0} \preceq \mathbf{u} \preceq \mathbf{1}\right\}
$$

2) canonical state variables $\mathbf{z}=\left(z_{1}, z_{2}, \ldots, z_{m}\right)^{T}$ :

$$
z_{i}=\frac{\psi_{i}-\psi_{i m}}{\psi_{i M}-\psi_{i m}}
$$

3) and generate $\mathbf{z}$ through a second-order criticallydamped differential equation

$$
\ddot{\mathbf{z}}+2 \boldsymbol{\alpha} \dot{\mathbf{z}}+\boldsymbol{\alpha}^{2} \mathbf{z}=\boldsymbol{\alpha}^{2} \mathbf{u}
$$

where $\boldsymbol{\alpha}^{2}=\operatorname{diag}\left(\left[\alpha_{1}^{2}, \alpha_{2}^{2}, \ldots, \alpha_{m}^{2}\right]\right)$ and $\alpha_{i}>0$ for $\forall i \in\{1,2, \ldots, m\}$.

Note that as long as the control inputs are admissible $\mathbf{u} \in$ $U,(11)$ and (13) would automatically ensure ${ }^{10}$ compatibility of $\psi$ with (10). This is to say that using (11)-(13) one can replace the original state inequality constraints $\psi \in \Psi$ with the canonical control constraints $\mathbf{u} \in U$. The question which remains however is how to include the new state variables $\mathbf{z}$ into the dynamics model (1). To this end we consider (12) to be a coordinate transformation that relates $\mathbf{z}$ with $\mathbf{q}$ and $\boldsymbol{\theta}$ through the following equality constraint

$$
\Psi(\mathbf{q}, \boldsymbol{\theta}, \mathbf{z})=\mathbf{0}
$$

where: $\boldsymbol{\Psi}(\mathbf{q}, \boldsymbol{\theta}, \mathbf{z})=\boldsymbol{\psi}(\mathbf{q}, \boldsymbol{\theta})-\operatorname{diag}(\mathbf{z})\left(\boldsymbol{\psi}_{M}(\boldsymbol{\theta})-\boldsymbol{\psi}_{m}(\boldsymbol{\theta})\right)+$ $\boldsymbol{\psi}_{m}(\boldsymbol{\theta})$. Under the assumption that $\partial \boldsymbol{\Psi} / \partial \boldsymbol{\theta} \in \mathbb{R}^{m \times m}$ is a full-rank matrix (i.e., $\forall(\mathbf{q}, \mathbf{z})$, $\operatorname{det}(\partial \boldsymbol{\Psi} / \partial \boldsymbol{\theta}) \neq 0$ ), one can invoke the implicit function theorem to (at least locally) define the solution of (14) with respect to $\boldsymbol{\theta}$,

$$
\boldsymbol{\theta}=\boldsymbol{\Psi}_{\theta}^{-1}(\mathbf{q}, \mathbf{z})
$$

where $\boldsymbol{\Psi}_{\theta}^{-1}$ is the corresponding inverse mapping of (14). The condition (i.e., $\forall(\mathbf{q}, \mathbf{z})$, $\operatorname{det}(\partial \boldsymbol{\Psi} / \partial \boldsymbol{\theta}) \neq 0$ ) for (15) to exist depends on the constraints. In this light, application of the proposed scheme may favor (although it is not restricted to) constraints that allow explicit computation of (15). This turns out to be the case for the model of the DLR Hand-Arm System used in Section IV.

\section{State-space representation of the dynamics}

Using (11), (13) and (15) we are now in position to explicitly embed state inequality constraints into the dynamics. To do this, let us consider a state-space representation of the

\footnotetext{
${ }^{9}$ The control inputs are piecewise continuous functions (i.e., $\mathbf{u} \in P C$ ) in the present consideration.

${ }^{10}$ Please note that (11) and (13) would also provide the necessary smoothness requirement on $\psi$ (i.e., $\psi \in \mathbb{C}^{2}$ ).
} 
link-side dynamics (1) and the canonical function generator (13), given as

$$
\dot{\mathbf{x}}=\mathbf{f}(\mathbf{x}, \mathbf{u}),
$$

where $\mathbf{x}=\left(\mathbf{x}_{1}^{T}, \mathbf{x}_{2}^{T}, \mathbf{x}_{3}^{T}, \mathbf{x}_{4}^{T}\right)^{T}=\left(\mathbf{q}_{1}^{T}, \dot{\mathbf{q}}_{1}^{T}, \mathbf{z}^{T}, \dot{\mathbf{z}}^{T}\right)^{T} \in$ $\mathbb{R}^{2(n+m)}$ is a state vector, while

$$
\mathbf{f}=\left[\begin{array}{c}
-\mathbf{M}^{-1}\left(\mathbf{x}_{1}\right)\left(\mathbf{C}\left(\mathbf{x}_{1}, \mathbf{x}_{2}\right) \mathbf{x}_{2}+\mathbf{G}\left(\mathbf{x}_{1}\right)-\mathbf{T}\left(\mathbf{x}_{1}, \mathbf{x}_{3}\right)\right) \\
\mathbf{x}_{4} \\
-2 \boldsymbol{\alpha} \mathbf{x}_{4}-\boldsymbol{\alpha}^{2} \mathbf{x}_{3}+\boldsymbol{\alpha}^{2} \mathbf{u}
\end{array}\right],
$$

is the corresponding vector field that includes the torque function expressed through the new canonical state variables defined by: $\mathbf{T}=\mathbf{T}\left(\mathbf{x}_{1}, \mathbf{x}_{3}\right)=\mathbf{T}(\mathbf{q}, \mathbf{z})=\boldsymbol{\tau}\left(\mathbf{q}, \mathbf{\Psi}_{\theta}^{-1}(\mathbf{q}, \mathbf{z})\right)$.

The parameter vector $\boldsymbol{\alpha}$ (containing one parameter for every embedded constraint) in (17) is inherited from (13). In mathematical terms, these parameter set the coupling strength between the dynamics and the control actions. Given the physical nature of the constraints, selection of these gains becomes intuitive. Moreover, under certain conditions, these gains can be used to directly embed the bandwidth limitations of the actuators into the formulation. This is detailed in the following.

1) Embedding range/bandwidth limitations on the motorside dynamics: Let us now assume that for a given actuator there is no limitation on the elastic deformation of the compliant elements, or more precisely, these limits (9) are not expected to be violated during the motion ${ }^{11}$. In this case, embedding all the constraints would mean that for $\forall \mathbf{u} \in U, \boldsymbol{\theta}_{m} \preceq \boldsymbol{\theta} \preceq \boldsymbol{\theta}_{M},|\dot{\boldsymbol{\theta}}| \preceq \dot{\boldsymbol{\theta}}_{M}$ are not violated by the motor trajectories planned by (16). We can enforce all these constraints by setting:

1) $\boldsymbol{\psi}=\boldsymbol{\theta}$; to directly embed the range limits (8), given by $\boldsymbol{\theta}_{m} \preceq \boldsymbol{\theta} \preceq \boldsymbol{\theta}_{M}$, into (17) and

2) any $\alpha_{i} \in\left(0, \alpha_{i M}\right]$ where $\alpha_{i M}=e \dot{\theta}_{i M} /\left(\theta_{i M}-\theta_{i m}\right)$ for $\forall i \in\{1,2, \ldots, m\}$; to satisfy the rate constraints ${ }^{12}$ (8) given by $|\dot{\boldsymbol{\theta}}| \preceq \dot{\boldsymbol{\theta}}_{M}$.

The first condition enables one to calculate the motor positions from the canonical states: $\boldsymbol{\theta}(t)=\operatorname{diag}\left(\mathbf{x}_{3}(t)\right)\left(\boldsymbol{\theta}_{M}-\right.$ $\left.\boldsymbol{\theta}_{m}\right)+\boldsymbol{\theta}_{m}$ (where $\mathbf{x}_{3}=\mathbf{z}$ ), while the second condition ensures that these trajectories are smooth enough to be precisely tracked by the real actuators (2).

2) Embedding deformation limits on the elastic elements: Let us now consider another case when the length of the springs in the actuators have to be constrained during the motion. In this case, we set: $\boldsymbol{\psi}=\boldsymbol{\phi}(\mathbf{q}, \boldsymbol{\theta})$ to directly embed the associated constraints (9) into (17). In physical terms this implies that the canonical states $\left(\mathbf{x}_{3}=\mathbf{z}\right)$ represent the normalized spring lengths, while the associated dynamics (third and fourth lines in (17)) defines the dynamics of the spring motion. Note that in this formulation, $\boldsymbol{\alpha}$ limits the bandwidth of the entire system (i.e., link-side and motorside dynamics), and as such it cannot be used to embed the

\footnotetext{
${ }^{11}$ This is indeed the case on our MACCEPA implementation, see Section IV.

${ }^{12}$ In practice, we often found $\alpha_{i M}$ conservative. This is mainly because $\alpha_{i M}$ is calculated assuming a maximal step response command $\theta_{i M}-\theta_{i m}$ although this may not be the usual command in applications. Due to this reason we often chose $\alpha_{i}>\alpha_{i M}$ as long as this does not jeopardize the tracking accuracy.
}

bandwidth limitations on the actuators solely. As alternative solution to this direct embedding, $|\dot{\boldsymbol{\theta}}| \preceq \dot{\boldsymbol{\theta}}_{M}$ could be treated as soft constraints and incorporated through penalty terms in the objective function. In Section IV we illustrate the viability of these formulations through simulation and practical application.

\section{Control task}

In this article, we consider the control tasks to be represented using the following formulation: For a given finite time interval $t \in[0, T]$, and for a given initial state of the system $\mathbf{x}(0)=\mathbf{x}_{0}$, find the admissible open-loop control inputs $\mathbf{u}=\mathbf{u}(t) \in U$ that minimizes the optimisation criterion:

$$
J=h(T, \mathbf{x}(T))+\int_{0}^{T} c(t, \mathbf{x}(t), \mathbf{u}(t, \mathbf{x}(t))) d t,
$$

where $J \in \mathbb{R}$ is the cost functional, $h(\mathbf{x}(T)) \in \mathbb{R}$ is the terminal cost, while $c(\mathbf{x}, \mathbf{u}) \in \mathbb{R}$ is the running cost used to encode the control objectives into the formulation [17].

\section{E. Solution methods}

Finding the optimal control inputs for non-linear plant dynamics (16) and non-quadratic cost $c(\mathbf{x}, \mathbf{u})$ is known to be non-trivial. Bellman's dynamic programming [18] and Pontryagins maximum principle (PMP) [19] provide general approaches to solve such problems. The numerical computation associated with direct application of these methods is however either computationally intractable for, high dimensional non-linear problems, or require sophisticated implementation to converge to the optimal solution [20]. Alternative methods that are computationally more tractable are: Differential Dynamic Programming (DDP) [21] and the Iterative Linear Quadratic Regulator/Gaussian (iLQR/G) framework [22]; that is the method of choice here.

1) $i L Q R$ : In the present paper we utilize the iLQR framework to devise the control sequence for the non-linear optimal control problem stated by (16) and (18). By means of the basic idea, the user defined initial control sequence and the corresponding state trajectories $\left(\mathbf{x}_{0}, \mathbf{u}_{0}\right)$ are iteratively improved using the solution of local LQR sub-problems. The sub-problems are formed by linear approximation of the system dynamics (16),

$$
\begin{gathered}
\delta \dot{\mathbf{x}}=\mathbf{A} \delta \mathbf{x}+\mathbf{B} \delta \mathbf{u}, \\
\mathbf{A}:=\frac{\partial \mathbf{f}}{\partial \mathbf{x}}=\left[\begin{array}{cccc}
\mathbf{0} & \mathbf{I}_{n \times n} & \mathbf{0} & \mathbf{0} \\
\frac{\partial \mathbf{f}_{2}}{\partial \mathbf{x}_{1}} & \frac{\partial \mathbf{f}_{2}}{\partial \mathbf{x}_{2}} & \frac{\partial \mathbf{f}_{2}}{\partial \mathbf{x}_{3}} & \mathbf{0} \\
\mathbf{0} & \mathbf{0} & \mathbf{I}_{m \times m} & \mathbf{0} \\
\mathbf{0} & \mathbf{0} & -\boldsymbol{\alpha}^{2} & -2 \boldsymbol{\alpha}
\end{array}\right], \mathbf{B}:=\frac{\partial \mathbf{f}}{\partial \mathbf{u}}=\left[\begin{array}{c}
\mathbf{0} \\
\mathbf{0} \\
\mathbf{0} \\
\boldsymbol{\alpha}^{2}
\end{array}\right],
\end{gathered}
$$

and quadratic approximation of the objective functional ${ }^{13}$ (18),

$$
\begin{array}{r}
\Delta J=\mathbf{h}_{x}^{T} \delta \mathbf{x}(T)+\delta \mathbf{x}^{T}(T) \mathbf{h}_{x x} \delta \mathbf{x}(T)+\int_{0}^{T}\left(\mathbf{c}_{x}^{T} \delta \mathbf{x}+\right. \\
\left.\mathbf{c}_{u}^{T} \delta \mathbf{u}+\delta \mathbf{x}^{T} \mathbf{c}_{x x} \delta \mathbf{x}+\delta \mathbf{x}^{T} \mathbf{c}_{x u} \delta \mathbf{u}+\delta \mathbf{u}^{T} \mathbf{c}_{u u} \delta \mathbf{u}\right) d t
\end{array}
$$

\footnotetext{
${ }^{13}$ In the linear approximation of the dynamics and the quadratic approximation of the objective functional, all quantities are evaluated at the current state and control sequence: $(\mathbf{x}, \mathbf{u})$.
} 
In every iteration, (19) and (20) is solved for $(\delta \mathbf{x}, \delta \mathbf{u})$ via a modified Ricatti-like system, and the new (improved) sequence is formed by $\mathbf{x} \leftarrow \mathbf{x}+\delta \mathbf{x}$ and $\mathbf{u} \leftarrow \mathbf{u}+\delta \mathbf{u}$. When the method converges (i.e., $\Delta J \approx 0$ achieved numerically), it provides the optimal state and control trajectories $\mathrm{x}^{*}=$ $\left(\mathbf{q}^{* T}, \dot{\mathbf{q}}^{* T}, \mathbf{z}^{* T}, \dot{\mathbf{z}}^{* T}\right)^{T}$ and $\mathbf{u}^{*} \in U$ with respect to the control problem (16) and (18).

2) The desired motor-side trajectories: In the present formulation the control inputs $\mathbf{u}(t)$ are abstract functions used for planning. Intermediate results of the planning are the canonical states $\mathbf{z}(t)=\mathbf{x}_{3}(t)$, while the final output is the motor side positions $\boldsymbol{\theta}^{*}=\boldsymbol{\Psi}_{\theta}^{-1}\left(\mathbf{q}^{*}(t), \mathbf{z}^{*}(t)\right)$. If the constraints on the rate of the actuators are incorporated into the formulation, the computed positions $\boldsymbol{\theta}^{*}$ will be smooth enough to be precisely tracked by the real actuators (2). This ensures that by using the computed solutions as optimal desired motor trajectories $\boldsymbol{\theta}_{d}=\boldsymbol{\theta}^{*}(t)$, the real motor positions $\boldsymbol{\theta}$ will closely follow the corresponding optimal motion $\boldsymbol{\theta}^{*}$. This argument holds independently of the specific dynamics of the actuators (2), i.e., this is a generic feature.

\section{Simulations AND EXPERIMENT}

In this section, we provide numerical simulation results and an experimental demonstration addressing the viability of the proposed optimal variable stiffness control formulation. The simulations/experiments involve two nonlinear variable stiffness systems: 1) the two-link variable stiffness arm [11] equipped with MACCEPA actuators [7] and 2) the integrated DLR Hand-Arm System (HASy) equipped with floating spring joints (FSJ) [1].

The reported results address a highly dynamic ballthrowing task. The objective function to be mimimized in this task is defined by,

$$
J=-d+\frac{1}{2} \int_{0}^{T}\left(w\|\mathbf{F}\|^{2}+\|\mathbf{u}\|_{\epsilon}^{2}\right) d t
$$

where $T$ is the time permitted for task execution ${ }^{14}, d=$ $d(\mathbf{q}(T), \dot{\mathbf{q}}(T))$ is the distance thrown, $\mathbf{F}=\mathbf{F}(\mathbf{q}, \boldsymbol{\theta})$ is the spring force $(\|*\|$ denotes the Euclidean norm), while $\|\mathbf{u}\|_{\epsilon}^{2}=\mathbf{u}^{T} \epsilon \mathbf{u}$ is a small positive regularisation term (i.e., $\boldsymbol{\epsilon} \approx 0$ ) that makes the objective explicitly control-dependent (and the control problem non-singular). Here we consider a dominantly distance maximization task by using a small effort weight $0<w \ll 1$.

\section{A. Optimal VS-control on a 2-link robot arm equipped with MACCEPA actuators}

Using the model of the two-link VS arm [11] (see Fig. 3), here we demonstrate in simulation, the effect of the actuator dynamics on the optimal solution. The purpose of this demonstration is to illustrate that the stiffness modulation strategy can significantly vary depending on the dynamics (bandwidth) of the VS actuator used to generate the behavior. It is obvious that having a limited stiffness range, the benefit

\footnotetext{
${ }^{14}$ While in the present paper the execution time $T$ is not optimised, this can be done within the present framework, using the method proposed in [23].
}

provided by variable-stiffness optimisation $\left(\theta_{3,4}=\theta_{3,4}(t)\right)$ compared to fixed-stiffness control (i.e., $\theta_{3,4}=$ const.) could be marginal. It is however also true that limitations on the bandwidth of the actuators can significantly alter the stiffness modulation strategy (and the overall performance).
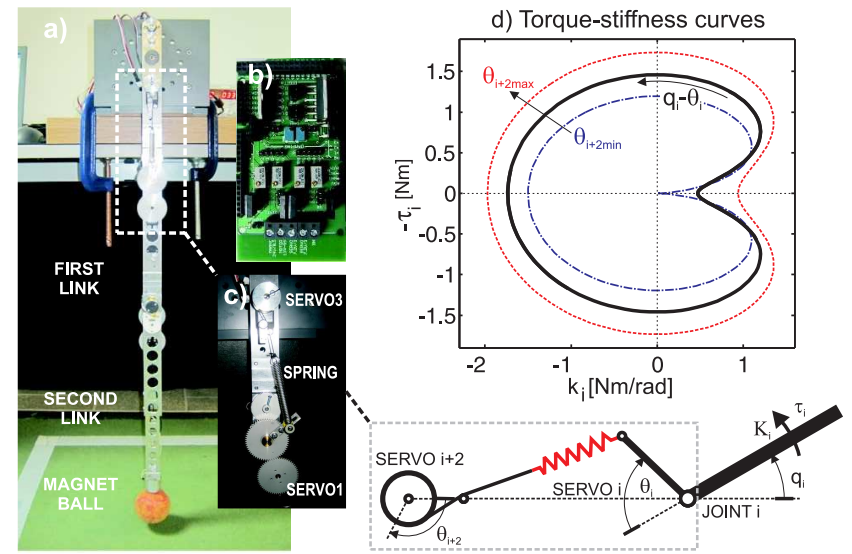

Fig. 3. a) Two-link variable-stiffness robot (with the tennis ball attached to the holding magnet), b) control board, c) variable-stiffness actuator, d) torque-stiffness curves characterizing the joints. Note that the three curves correspond to the minimal, mid-range and maximal position of the stiffness-adjusters $\theta_{3,4}$ respectively. Each of these curves define the passive torque stiffness characteristic of the joint under constant pretension $\theta_{3,4}=$ const. By changing the set-point commands $\theta_{1,2}$, but fixing the pretension commands $\theta_{3,4}=$ const., one can move along these passive isocurves in the torque-stiffness plane. However by changing the pretensioning continuously in time $\theta_{3,4}=\theta_{3,4}(t)$, one can also modify these curves during the movement. This ability to modify the passive joint characteristics allows simultaneous torque and stiffness variation around the joints.

In Fig. 4 we show that using fast (high-bandwidth) actuators, $\alpha_{3,4}=[\sqrt{1000}, \sqrt{1000}]$ instead of slow (lowbandwidth) ones $\alpha_{3,4}=[\sqrt{20}, \sqrt{20}]$, not only affects the systems performance but it can qualitatively modify the stiffness control strategy. Indeed, when the actuators are slow, active modulation of the torque-stiffness characteristics of the joints appears to be not beneficial (see gray lines in Fig. 4d), as opposed to the case when the same task is realised with actuators capable of fast stiffness adjustments (black lines in Fig. 4d). Moreover, there is an obvious danger that the actuator dynamics (when slow) filters out any taskspecific stiffness modulation, but also that some actuatorspecific stiffness control feature are (mistakenly) attributed as task induced. Clarity and insights into these issues can guide new VS actuator designs, but are also highly relevant when interpreting human stiffness (impedance) control strategies that are subject to realistic biological (e.g. muscle tendon) bandwidth-limitations [24]. In addition to the present simulation result, this two-link VS arm (Fig. 3) was used in a simulation study on variable stiffness brachiation [25] as well as a hardware realisation of a variable stiffness throwing experiments [11]. The reader is referred to this latter work for a detailed comparison of performance benefits (measured by distance thrown) provided by variable stiffness optimisation (as opposed to an optimal but fixed stiffness control). 

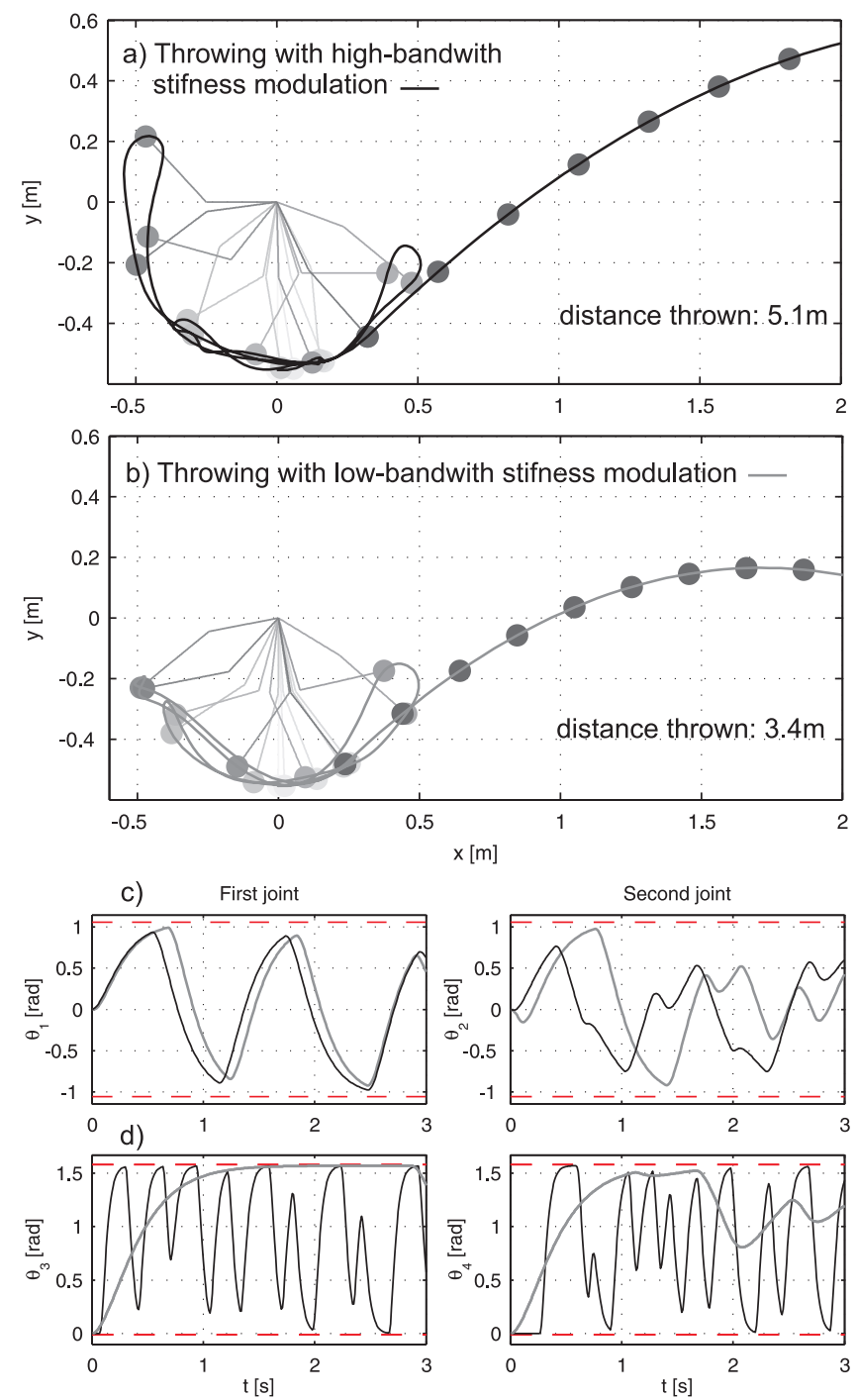

Fig. 4. Ball-throwing with high and low bandwidth actuators. Stroboscopic view of the throwing motion: a) variable-stiffness control using high-bandwidth (fast) stiffness adjustments: $\boldsymbol{\alpha}=$ $\operatorname{diag}([\sqrt{50}, \sqrt{50}, \sqrt{1000}, \sqrt{1000}])$, results plotted in black and b) optimal control for the same task while using low-bandwidth (slow) stiffness adjustments: $\boldsymbol{\alpha}=\operatorname{diag}([\sqrt{50}, \sqrt{50}, \sqrt{20}, \sqrt{20}])$, results plotted in gray. c) Optimal set-point command positions $\theta_{1,2}$,d) optimal stiffness adjuster positions $\theta_{3,4}$. The arm is initialised from a vertical resting configuration. Limits on the motor positions (depicted with dashed red lines) are set to: $\theta_{1,2} \in[-\pi / 3, \pi / 3] \mathrm{rad}$ and $\theta_{3,4} \in[-\pi / 2, \pi / 2] \mathrm{rad}$.

\section{B. Optimal VS-control with the DLR HASy arm}

The DLR integrated Hand-Arm System (HASy), depicted in Fig. 1, is a state-of-the-art variable stiffness robot resembling the complexity of a human upper limb. Mechanically, this device has 26 kinematic DOFs and 52 actuators. All the joints of the robot are actuated through complex variable stiffness mechanisms. The presented simulations/experiments are executed in the sagittal plane, namely the shoulder and elbow rotation joint. By design, these are Floating Spring Joints (FSJs) depicted in Fig. 5, see [6]. In these FSJs, the VS mechanism involves two main motors that modulate the set-point of the virtual springs (i.e., $\theta_{1,2}$ ) and two small motors that adjust the joint stiffness (i.e., $\theta_{3,4}=\sigma_{1,2}$ ) see
Fig. 5b,d. Using servo control (see Section II-A (3)) for these four motor positions $\boldsymbol{\theta}=\left[\theta_{1}, \theta_{2}, \sigma_{1}, \sigma_{2}\right]^{T}$, one can perform simultaneous torque and stiffness modulation.

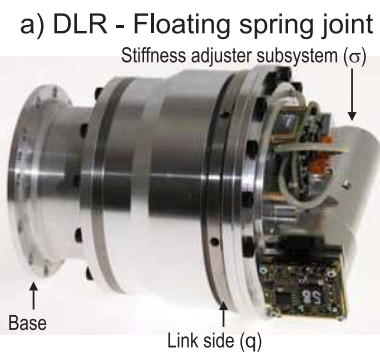

b) Torque-stiffness curves

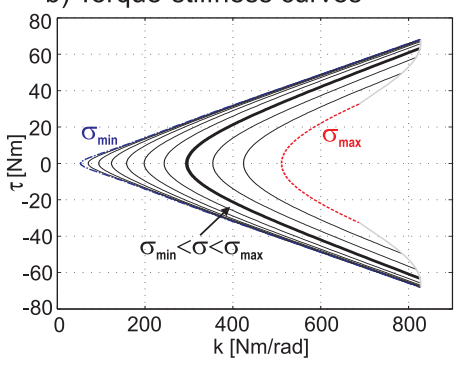

c) Internal cam-disc mechanism

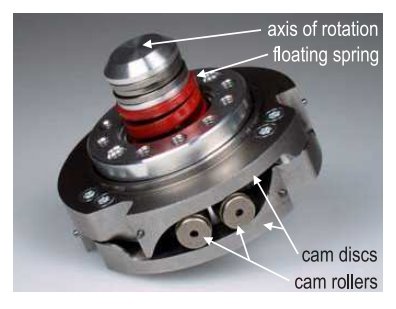

d) Schematic representation

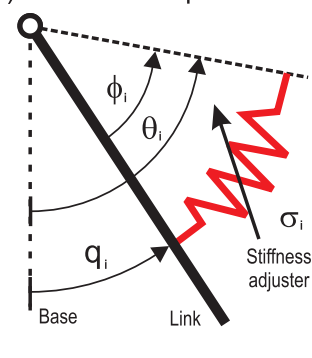

Fig. 5. The DLR floating spring joint. For more details on design the reader is refered to [1]

1) Specification of the constraints: The state/rate constraints associated with the limitations on the position and velocities of the stiffness adjuster motors $\sigma$ and the deformation limits on the floating springs are given as:

$$
\begin{array}{r}
\mathbf{0} \preceq \boldsymbol{\sigma} \preceq \boldsymbol{\sigma}_{M},|\dot{\boldsymbol{\sigma}}| \preceq \dot{\boldsymbol{\sigma}}_{M}, \\
\boldsymbol{\phi}_{m}(\boldsymbol{\sigma}) \preceq \boldsymbol{\phi} \preceq \boldsymbol{\phi}_{M}(\boldsymbol{\sigma}),
\end{array}
$$

where the maximal limits are defined by: $\sigma_{M}=$ $0.178[1,1]^{T} \mathrm{rad}, \dot{\boldsymbol{\sigma}}_{M}=0.541[1,1]^{T} \mathrm{rad} / \mathrm{s}$ and $\phi_{m}(\boldsymbol{\sigma})$ and $\phi_{M}(\boldsymbol{\sigma})$ are the state dependent constraint boundaries depicted in Fig. 6 (dashed red lines). The present formulation encodes these as hard constraints.

Additional limits to be considered are the range/rate limits associated with the main motors (i.e., that modulate the setpoint of the virtual joint springs $\theta_{1,2}$, see Fig. 5d) These constraints: $\theta_{1,2 m} \leq \theta_{1,2} \leq \theta_{1,2 M},\left|\dot{\theta}_{1,2}\right| \leq \dot{\theta}_{1,2 M}$, where $\theta_{1,2 m}=[-1.05,-0.44]^{T} \mathrm{rad}, \theta_{1,2 M}=[3.05,2.27]^{T} \mathrm{rad}$ and $\dot{\theta}_{1,2 M}=8[1,1]^{T} \mathrm{rad} / \mathrm{s}$ define the maximal ranges, are treated as soft-constraints and embedded through penalty terms in the cost function (experimental results verified that these constraints were indeed satisfied consistently).

2) Simulation result: In Fig. 6 simulation results are presented demonstrating the treatment of the complex state inequality constraints given by (22). For this purpose, we report a typical result obtained using optimal variable stiffness control $\boldsymbol{\sigma}=\boldsymbol{\sigma}(t)$ and optimal fixed stiffness control - in the present setup, the minimum stiffness $\boldsymbol{\sigma}(t)=\mathbf{0}$ was computed as the optimal fixed value; this is intuitive since it provides the highest torque range on the DLR FSJ (see Fig. 5b). In Figs. $6 \mathrm{c}, \mathrm{d}$ we show that the developed formulation enforces the state inequality constraints regardless of whether fixed or variable stiffness control is considered. Fig. 6e shows 

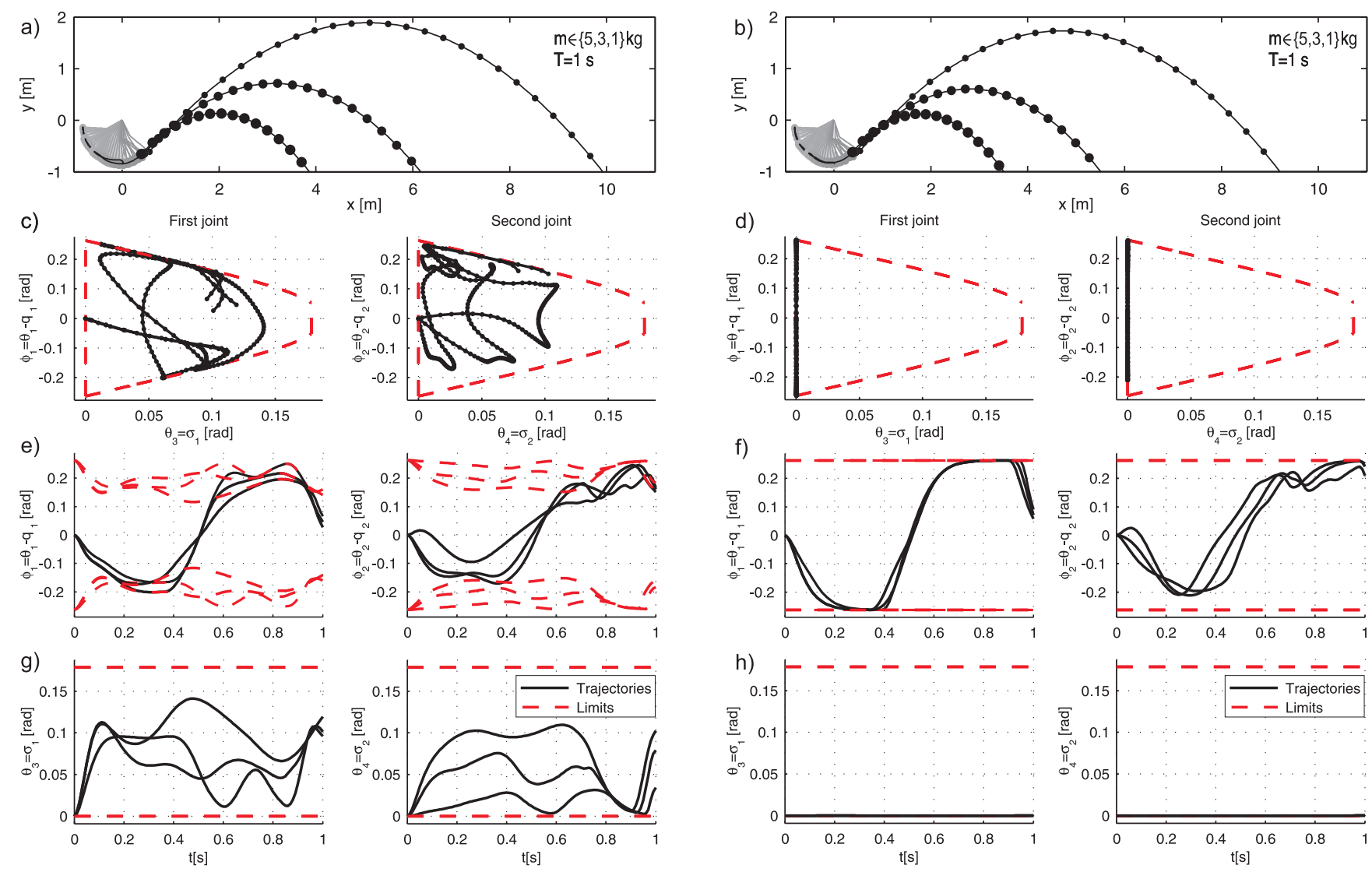

Fig. 6. Treatment of constraints: a) optimal VS control, b) optimal fixed stiffness control. The simulations are performed with different ball masses: $m \in\{1,3,5\} \mathrm{kg}$ and fixed execution time: $T=1 \mathrm{~s}$. The limits on $\phi$ are depicted with dashed red lines in Fig. $6 \mathrm{c}$-f while the constraints on $\theta_{3,4}=\sigma_{1,2} \in$ $[0,0.178] \mathrm{rad}$ are shown in Fig. 6g,h. In addition, we set: $\theta_{1} \in[-1.05,3.05] \mathrm{rad}, \theta_{2} \in[2.27,0.44] \mathrm{rad}, \dot{\theta}_{1,2} \in[-8,8] \mathrm{rad} / \mathrm{s}$ and $\boldsymbol{\alpha}=25 \mathrm{diag}([1,1,1,1])$ in these simulations. In all cases, the throwing motion is started from a vertical hanging configuration with minimum stiffness. Note that in Figs. $6 \mathrm{c}-\mathrm{f}$ each solution (black lines) strictly satisfies its own corresponding constraint (red dashed lines).

that this is nontrivial since the boundaries of the constraints depend on stiffness modulation (e.g., higher $\sigma$ in Fig. $6 \mathrm{~g}$ result in stronger restriction on $\phi$ in Fig. 6e) and as such they change through time. In Figs. 6e,f one can recognize a bangbang like strategy (especially on the first joint) that is smooth (due to the bandwidth limitations of the actuators) and is planned under changing constraint boundaries (Fig. 6e).

3) Hardware Experiment: In Fig. 7 we present a ball throwing experiment using our variable stiffness optimisation and control methodology on the DLR HASy. For the experimental video, the reader is referred to the multimedia material (http://goo.gl/aQ5MP). Fig. 7a shows a characteristic counter movement action that is a general feature of ball throwing for humans. An excellent match between the simulated and the real link-side motion $\mathbf{q}(t)$ can be see in Fig. 7b. This is partly because of an accurate system model, but also because the proposed optimisation algorithm plans motor trajectories that respects all physical constraints and as such could be precisely tracked on this complex system (see $\boldsymbol{\theta}(t)$ in Figs. 7b,c). Fig. 7c shows a similar stiffness variation strategy seen in the simulations (Fig. $6 \mathrm{~g}$ ) - the decrease and subsequent rapid increase of the stiffness just before the ball release $(t=1 s)$. The effect of this strategy is a rapid acceleration of the arm at the end of the movement that contributes to improved task performance (distance thrown). In Fig. 7d, we see a characteristic sequential velocity peak, first on the proximal link $\dot{q}_{1} \approx 6 \mathrm{rad} / \mathrm{s}$ and then on the distal link $\dot{q}_{1} \approx 5.2 \mathrm{rad} / \mathrm{s}$. Note that these velocity peaks could have not been achieved with rigid actuators (where $\dot{\mathbf{q}}=\left[\dot{\theta}_{1}, \dot{\theta}_{2}\right]^{T}$ ) under the velocity limit $\dot{\theta}_{1,2} \leq 3 \mathrm{rad} / \mathrm{s}$ used here. In terms of task performance, using kinematic calculations, we found that while a rigid robot in the same setup could ideally throw a ball $d=3.6 m$, the VS joints allowed the DLR arm to throw a ball $d \approx 5 m$ (verified by experiments).

\section{Conclusion}

In this paper, we provide an optimal-control formulation for state constrained variable stiffness control. Instead of using complex numerical algorithms for the treatment of the constraints, this formulation allows one to explicitly embed state inequality constraints into the dynamics using control limits. In practice, this formulation can be used to embed actuator bandwidth limitations and/or deformation limits on the elastic elements inherent to arbitrary VSA designs. Here, we show the viability of the proposed formulation on two conceptually different variable-stiffness systems performing a ball-throwing task, first in simulations and then, in an experiment realised with the DLR Hand-Arm System. 

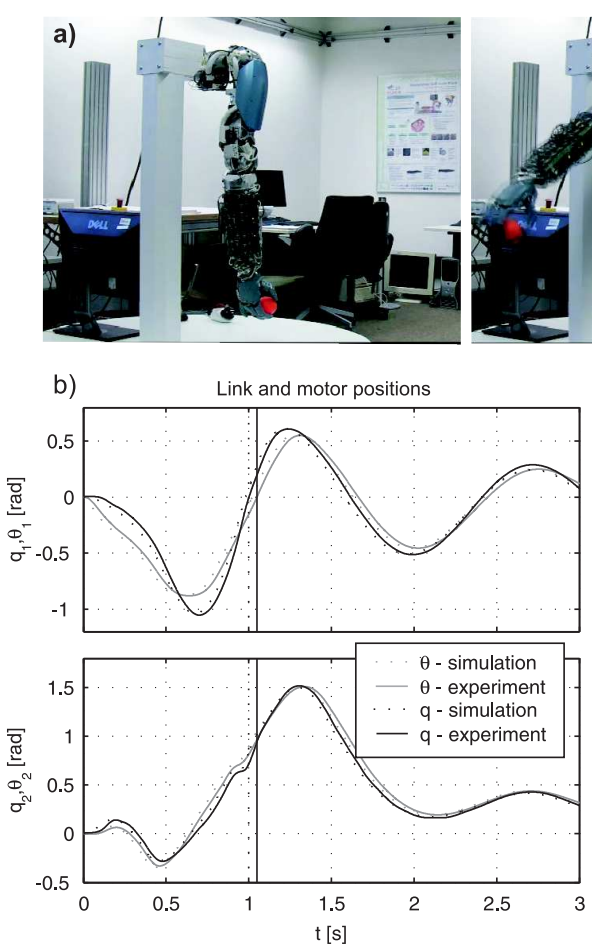

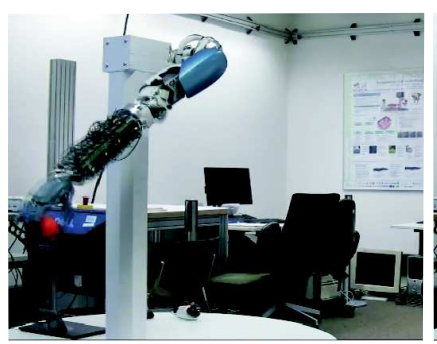

c)
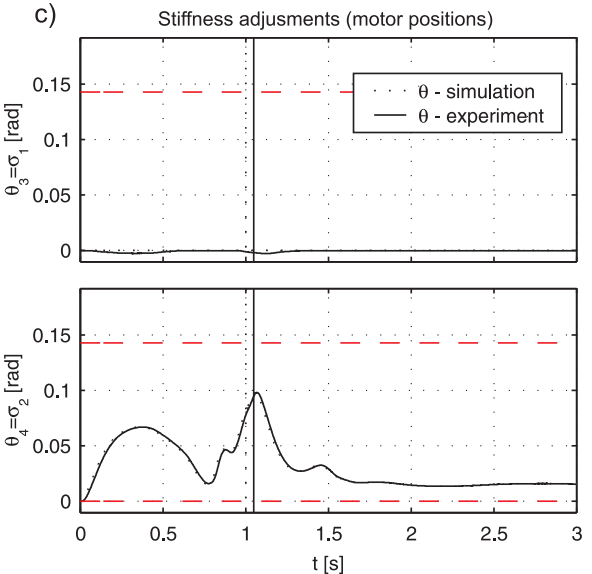
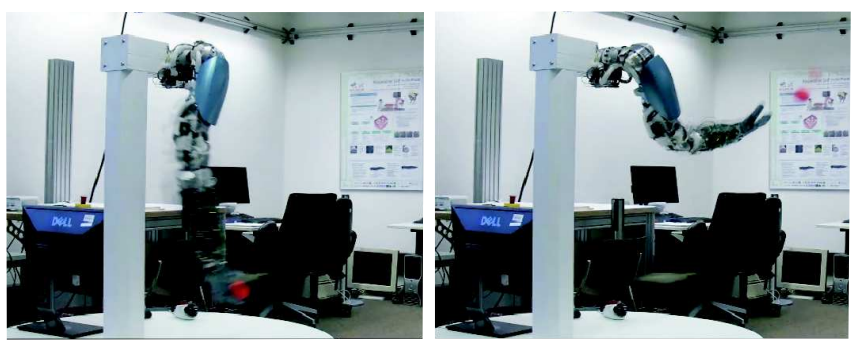

d)
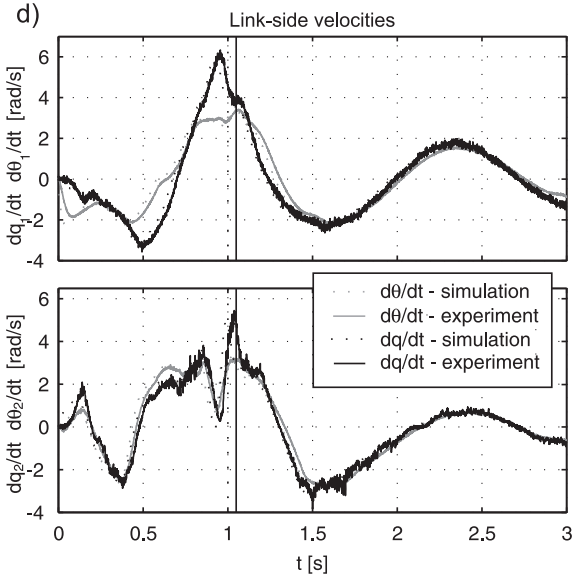

Fig. 7. Ball-throwing using optimal variable stiffness control: a) motion sequence of the experiment, b) motor and link-side trajectories, c) stiffness adjustment, d) motor and link-side velocities. The experiment is performed with a ball weighting $m=0.06 \mathrm{~kg}$ and fixed execution time: $T=1 \mathrm{~s}$. The limits are set to: $\theta_{1} \in[-1.05,3.05] \mathrm{rad}, \theta_{2} \in[2.27,0.44] \mathrm{rad}, \theta_{1,2} \in[-3,3] \mathrm{rad} / \mathrm{s}, \theta_{3,4}=\sigma_{1,2} \in 0.8[0,0.178] \mathrm{rad}$ and $\boldsymbol{\alpha}=\operatorname{diag}([25,25,15,15])$. The throwing motion is started from a vertical hanging configuration with no initial velocity and minimum stiffness.

\section{ACKNOWLEDGEMEnTs}

This work is supported by EU FP7 grants STIFF and VIACTORS. The authors acknowledge the contribution of the DLR Hand-Arm System hardware design team.

\section{REFERENCES}

[1] M. Grebenstein et.al., "The DLR Hand Arm System," in IEEE ICRA, (Shanghai, China), pp. 3175-3182, 2011.

[2] N. Hogan, "Impedance control: An approach to manipulation," ASME J. Dyn. Sys., Meas., Control, vol. 107, pp. 1-24, 1985.

[3] K. Koganezawa, Y. Watanabe, and N. Shimizu, "Antagonistic musclelike actuator and its application to multi-dof forearm prosthesis," Advanced Robotics, vol. 12, no. 7-8, pp. 771-789, 1999.

[4] G. Tonietti, R. Schiavi, and A. Bicchi, "Design and control of a variable stiffness actuator for safe and fast physical human/robot interaction," in IEEE ICRA, (Barcelona, Spain), pp. 526-531, 2005.

[5] S. A. Migliore, E. A. Brown, and S. P. DeWeerth, "Novel nonlinear elastic actuators for passively controlling robotic joint compliance," Journal of Mechanical Design, vol. 129, no. 4, pp. 406-412, 2007.

[6] S. Wolf and G. Hirzinger, "A new variable stiffness design: Matching requirements of the next robot generation," in IEEE ICRA, (Pasadena, CA, USA), pp. 1741-1746, 2008.

[7] R. van Ham et. al., "MACCEPA, the mechanically adjustable compliance and controllable equilibrium position actuator: Design and implementation in a biped robot.," Robotics and Autonomous Systems, vol. 55, no. 10, pp. 761-768, 2007.

[8] J. W. Hurst, J. Chestnutt, and A. A. Rizzi, "The actuator with mechanically adjustable series compliance," IEEE Transactions on Robotics, vol. 26, no. 4, pp. 597-606, 2010.

[9] D. J. Braun, M. Howard, and S. Vijayakumar, "Exploiting variable stiffness in explosive movement tasks," in Robotics: Science and Systems, (Los Angeles, USA), 2011.

[10] S. Haddadin, M. Weis, S. Wolf, and A. Albu-Schäffer, "Optimal control for maximizing link velocity of robotic variable stiffness joints," in IFAC World Congress, (Milano, Italy), 2011.
[11] D. J. Braun, M. Howard, and S. Vijayakumar, "Optimal variable stiffness control: Formulation and application to explosive movement tasks," Autonomous Robots: DOI 10.1007/s10514-012-9302-3, 2012.

[12] S. Haddadin, F. Huber, and A. Albu-Schäffer, "Optimal control for exploiting the natural dynamics of variable stiffness robots," in IEEE ICRA, 2012.

[13] K. J. Salisbury, "Active stiffness control of a manipulator in cartesian coordinates," in IEEE CDC, pp. 383-388, 1980.

[14] M.Spong, "Modeling and Control of Elastic Joint Robots," ASME J. Dyn. Sys., Meas., Control, vol. 109, no. 4, pp. 310-319, 1987.

[15] H. Goldstein, Classical Mechanics. Addison-Wesley, 1980.

[16] F. A. Mussa-Ivaldi, N. Hogan, and E. Bizzi, "Neural, mechanical, and geometric factors subserving arm posture in humans," Journal of Neuroscience, vol. 5, pp. 2732-2743, 1985.

[17] W. L. Nelson, "Physical principles for economies of skilled movements," Biological Cybernetics, vol. 46, no. 2, pp. 135-147, 1983.

[18] R. Bellman, Dynamic Programming. Princeton University Press, 1957.

[19] L. S. Pontryagin, V. G. Boltyanskii, R. V. Gamkrelidze, and E. F. Mishchenko, The Mathematical Theory of Optimal Processes. John Wiley and Sons, New York, 1962.

[20] J. T. Betts, "Survey of numerical methods for trajectory optimization," AIAA Journal of Guidance, Control and Dynamics, vol. 21, no. 2, pp. 193-207, 1998.

[21] D. H. Jacobson and D. Q. Mayne, Differential Dynamic Programming. New York: Elsevier, 1970.

[22] W. Li and E. Todorov, "Iterative linearization methods for approximately optimal control and estimation of non-linear stochastic system," International Journal of Control, vol. 80, no. 9, pp. 1439-1453, 2007.

[23] K. Rawlik, M. Toussaint, and S. Vijayakumar, "An approximate inference approach to temporal optimization in optimal control," in NIPS, (Vancouver, Canada), 2010.

[24] J. M. Winters and L. Stark, "Analysis of fundamental human movement patterns through the use of in-depth antagonistic muscle models," IEEE Transactions on Biomedical Engineering, vol. BME-32, pp. 826839, 1985.

[25] J. Nakanishi and S. Vijayakumar, "Exploiting passive dynamics with variable stiffness actuation in robot brachiation," in Robotics: Science and Systems, (Sydney, Australia), 2012. 